\section{Prognostischer Wert der MRD bei AML}

\author{
Bei verschiedenen Leukämien nutzen Ärzte bereits den prognostischen \\ Wert der minimalen Resterkrankung (MRD) nach einer Induktionstherapie. \\ Französische Ärzte haben untersucht, ob dies auch bei der akuten \\ myeloischen Leukämie (AML) eine hilfreiche Option sein könnte.
}

M utationen des Nucleophosmin1-Gens (NPM1m) könnten bei der AML ein attraktives Target für das MRDMonitoring sein. In einigen Studien zeigte sich eine gute Korrelation zwischen dem früh detektierten NPM1m-MRDLevel in Blut oder Knochenmark und dem klinischen Ergebnis der Patienten. Nun wurde untersucht, ob die NPM1mMRD-Spiegel auch Grundlage der Entscheidung für eine allogene Stammzelltransplantation (alloSCT) sein können.

Bei 152 Patienten der ALFA-0702-Studie mit NMP1m war eine MRD-Evaluation in erster Remission verfügbar. Patienten mit ungünstiger Prognose laut ELN-Klassifikation kamen für eine alloSCT infrage. Patienten mit einem Absinken der NPM1m-MRD im peripheren
Blut von $<4$ Log-Stufen hatten eine höhere kumulative Inzidenz für ein Rezidiv (Subhazard Ratio 5,83; p < 0,001) und ein kürzeres Gesamtüberleben (OS; Hazard Ratio [HR] 10,99; $\mathrm{p}<0,001)$ als Patienten mit einer Abnahme um $\geq 4$ Log-Stufen.

Multivariat waren ein abnormaler Karyotyp, eine FLT3-internal Tandem Duplikation (ITD) und eine Reduktion der NPM1m-MRD-Spiegel um $<4$ Log-Stufen signifikant mit einer höheren Rezidivinzidenz und einem kürzeren OS verbunden. Bei Patienten mit FLT3-ITD blieben das Alter, die Leukozytenzahl und die Reduktion der NPM1m-MRD-Spiegel prognostisch signifikant, nicht aber das allelische Verhältnis der FLT3-ITD. Das klinische Ergebnis von Patienten mit einer geringen NPM1m-MRD-Reduktion im peripheren Blut konnte durch eine alloSCT erheblich verbessert werden (HR 0,$25 ; \mathrm{p}=0,047$ für krankheitsfreie Überleben [DFS] und für OS). Der Nutzen einer alloSCT konnte jedoch nicht bei Patienten mit einer NPM1m-MRD-Reduktion $\mathrm{um} \geq 4$ Log-Stufen beobachtet werden (DFS: HR 1,62; $\mathrm{p}=0,42$; OS: HR 2,11; $\mathrm{p}=0,26)$. In beiden Analysen zeigte sich eine signifikante Interaktion zwischen alloSCT-Effekt und MRD-Ansprechen (DFS: $\mathrm{p}=0,024 ;$ OS: $\mathrm{p}=0,027$ ).

Fazit: Die Daten stützen die prognostische Bedeutung der NPM1m-MRD-Spiegel im peripheren Blut nach einer Induktionstherapie, unabhängig vom zytogenetischen und molekularen Kontext. Der NPM1m-MRD-Spiegel könnte auch als prädiktiver Faktor für die Indikation zur alloSCT genutzt werden. Christina Berndt

Balsat M et al. Postinduction Minimal Residual Disease Predicts Outcome and Benefit From Allogeneic Stem Cell Transplantation in Acute Myeloid Leukemia With NPM1 Mutation: A Study by the Acute Leukemia French Association Group. J Clin Oncol. 2017;35(2):185-93.

\title{
Ist Abwarten beim FL noch möglich?
}

\section{Früher war "watch and wait" eine anerkannte Strategie bei Patienten mit follikulärem Lymphom (FL). Aber kann abwartendes Beobachten noch eine Option sein, seit es den potenten Antikörper Rituximab gibt? Eine retrospektive Analyse aus Japan gibt Antwort.}

$B^{\mathrm{c}}$ evor Rituximab verfügbar war, war ,watch and wait" eine anerkannte Strategie. Mehrere Studien hatten ergeben, dass das Abwarten und Beobachten das klinische Ergebnis von FL-Patienten unabhängig von ihrer Tumorlast nicht beeinträchtigte. Mit Rituximab ist nun aber eine potente Behandlungsoption vorhanden. Ist die Watch-and-WaitStrategie damit überholt? Japanische Kollegen sind der Frage nachgegangen.

Retrospektiv wurden 348 Patienten analysiert (18-85 Jahre), bei denen in den Jahren 2000 bis 2012 ein FL diagnostiziert worden war. 101 Patienten wurden zunächst nur beobachtet, 247 wurden sofort behandelt. Wann die Erstbehandlung begonnen wurde, blieb den Ärzten überlassen. Dabei wurde aufgrund pathologischer und/oder klinischer Faktoren entschieden, ob von „watch and wait“"zu einer Behandlung übergegangen wurde. Von einer klinischen Transformation der Erkrankung hin zum dringend behandlungsbedürftigen Zustand wurde z. B. dann ausgegangen, wenn der Lactatdehydrogenase-Spiegel des Patienten stark anstieg, der Tumor stark wuchs oder eine Hyperkalzämie auftrat.

Das Follow-up dauerte im Mittel 75 Monate. Die geschätzte mittlere Zeit bis zum Therapieversagen (TTF) unter den Patienten, bei denen eine Behandlung erst auf die Watch-and-Wait-Strategie folgte, betrug 92 Monate; die TTF in der Kohorte mit sofortiger Behandlung lag dagegen bei 77 Monaten. Dieser Unterschied war nicht signifikant ( $\mathrm{p}=0,272)$.
Einer Multivarianzanalyse zufolge war das Tumorstadium ein wesentlicher prädiktiver Faktor für die TTF (Hazard Ratio [HR] 1,19; p < 0,05). Weder die Gesamtüberlebensrate noch das kumulative Risiko einer Transformation unterschieden sich zwischen der Watch-andWait-Kohorte und der Kohorte mit sofortiger Behandlung signifikant.

Fazit: Reines Abwarten hat dieser Arbeit zufolge keinen negativen Einfluss auf die TTF, das Gesamtüberleben oder die Transformation bei ausgewählten FLPatienten. Diese Ergebnisse legen nahe, dass ein abwartendes Beobachten trotz der Verfügbarkeit von Rituximab immer noch eine akzeptable Strategie ist. Sinnvoll wäre eine prospektive Kohortenstudie, inklusive einer Evaluierung optimaler Kriterien dafür, wann eine AntiLymphom-Behandlung zu beginnen ist.

Christina Berndt

Yuda S et al. Influence of the watch and wait strategy on clinical outcomes of patients with follicular lymphoma in the rituximab era. Ann Hematol. 2016;95(12):2017-22. 